\title{
Weather indices of agricultural production in the Netherlands 1948-1989. 1. General methodology and the arable sector
}

\author{
A. J. OSKAM \\ Department of Agricultural Economics, Wageningen Agricultural University, P.O. Box \\ 8130, NL 6700 EW Wageningen, Netherlands
}

Received 15 January 1991; accepted 10 May 1991

\section{Summary}

This paper starts with the methodology of deriving weather indices for different types of agricultural production. These indices should be useful for economic research. The methodology applied to the arable sector covers the period 1948-1989. Twelve products are included. At an aggregate level most extreme variations in weather indices are less than $23 \%$. in the long term, systematic developments of yields are more important than weather although there are substantial differences between individual crops. Average yield increase per year is $1.3 \%$. The annual standard deviation due to weather is $6 \%$. Although the influence of weather is slightly decreasing in a relative sense, the levels of yield fluctuations are still increasing.

Keywords: weather indices, arable crops, yield fluctuations

\section{Introduction and general methodology}

For several types of research, the availability of an indicator about past weather conditions can be very useful. We will concentrate on the need for weather indices in economic research. A weather index is an aggregate quantitative indicator of weather conditions. For different types of research, however, different indicators may be relevant. Here we will direct our attention to quantitative economic research, using annual observations of output(s), inputs, prices and technology. Weather can be an important disturbing factor in explaining relations between these variables. More specific examples of the usefulness of weather indices are:

1. Productivily analysis and the measurement of technological change. Because productivity measures and indicators of technological change are highly influenced by weather conditions, productivity measures over short periods are unreliable. Extreme weather conditions at the beginning and end of even longer periods have an influence on productivity measures (Oskam, 1991). Therefore, a relevant weather index can improve considerably the measurement of technological change. 
2. Estimation of production functions. Here the same arguments used for technological change can be applied. The estimated coefficients of a production function can be influenced by weather conditions. When input levels are independent of the weather, estimated coefficients of input variables will be unbiased. Yet, the uncertainty of coefficients can be still much higher without a variable representing changes of weather conditions. Moreover, the coefficient of the technology variable(s) can be biased and is less reliable without adding a weather index in the estimation procedure.

3. Estimating supply functions for agricultural outputs and/or demand relations for agricultural inputs. Supply functions give the relation between quantity of supply and output prices, input prices and technology; this type of analysis is called supply analysis. Here the same reasoning used for production functions is applicable. Time-series cross-section analysis for relative short periods, in particular, has an urgent need to eliminate the effects of fluctuating weather conditions (Thijssen, 1989).

4. In general, every agricultural sector model can be improved by adding reliable weather indices as exogenous variables (Cromarty, 1959; Oskam, 1987; Oskam et al., 1989).

These reasons are sufficient to look for reliable weather indices at the level of specific crops, subsectors of the agricultural sector or the whole sector.

Several approaches can be used. A good overview of methodology deriving weather indices has been given by Stallings (1960, 1961), Shaw (1964) and Doll (1967). The applicability of a particular approach depends also on the availability of data. Here we will give attention to three different methods to construct weather indexes, bearing in mind that an ideal weather index for a particular function consists of an index variable, which contains all the direct and indirect weather effects influencing this function. Direct effects have a direct influence on the particular function. Indirect effects result from adjustments as in the use of inputs due to different weather conditions. An example can be instructive. Assume that above some minimum level, rainfall during the growing season of sugar beets has no effect on the sugar production per hectare, while sunshine has a positive effect. The particular weather index describing the production of sugar per hectare will be influenced by sunshine and by rain up to some level. Such an index variable can be used to explain productivity or production per hectare and also for supply analysis. However, describing the number of working hours and machinery hours per unit of product, rain may have a negative effect, while sunshine is unimportant unless rainfall is high. A relevant weather index for the description of the number of working hours per unit of sugar differs from the previous one. This implies that even a constructed weather index for a particular product can be used only selectively. Although different weather indexes might be useful, we will construct one index only to simplify matters.

Literature on constructing weather indexes or incorporating weather influences outlines the following main lines:

1. Constructing a particular index by means of weather variables such as temperature, rainfall, and sunshine or more direct variables such as soil moisture and 
radiation. Here the period of the year can also be a very important variable. The particular relation between weather variables and the resulting yield level will be estimated by means of regression analysis (Doll, 1967). A related method is to incorporate directly weather variables in an economic model (Oury, 1965, 1966) that allows to include interaction between weather variables and 'economic' variables.

2. Using the yield levels of variety trials and other experimental fields. A direct construction of weather indices can be applied. When these fields are operated under constant conditions, all differences arise from weather (Stallings, 1960, 1961). Sample fields often have different objectives, and this implies a particular development in yields that can be represented by trend variables (Stallings) or by using moving averages (Shaw, 1964). When data of field experiments are only available for a part of the period, a relation between yields and weather variables can be estimated. A functional relation that will be constant in time can be extrapolated.

3. Dividing time series of revenues in a systematic and a stochastic part. By assumption, the stochastic part has been caused by weather variation (and related variables, such as diseases and plagues), while the systematic part is related to technical development and systematic (economic) factors.

Each method has a number of difficulties. However, data availability and main factors influencing quantity variation determine which method is most useful. For the arable sector method 3 will be used. In a consecutive paper for grassland methods 2 and 3 are applied (Oskam \& Reinhard, 1992). Each method will be explained before its application with special emphasis given to difficulties and biases of each method (see Sections 'Methodology' and 'Discussion'). After the presentation of the empirical results the resulting systematic parts and weather indices will be discussed.

\section{Methodology of constructing weather indices for arable products and the arable sec- tor}

This method uses average annual yield data for particular arable products. The main assumption is that variation in yields originates from two different sources:

- A systematic part containing all variables such as technical development, use of inputs, increasing of decreasing area with differences in potential yield, etc.

- A stochastic part, mainly caused by variation of the weather among the different years.

Because data of average yields of arable crops are readily available, this method is easy to apply.

Here we assume that the yield per hectare $(Y)$ for a particular arable product depends on a weather index $(W)$ and a vector of other variables $(x)$ :

$$
Y=\mathrm{f}(W, x)
$$

Under the assumption that the function is separable in $W$ and $x$ and $\mathrm{f}$ is linear homogenous in $\mathrm{W}$, the following holds (Chambers, 1988): 


$$
Y=W \cdot \mathrm{f}(1, x)=W \mathrm{f}_{1}(x)
$$

Here $f_{1}(x)$ is the systematic part of the yield while $W$ is the stochastic part. This specification implies a multiplicative model with a higher yield variation for increasing systematic yields. Next, we make the assumption that the systematic part can be represented by an exponential polynomial trend function:

$$
Y=e^{\mathrm{g}(T)} \cdot W
$$

Where $\mathrm{g}(T)$ is a polynomial trend function. A logarithmic transformation of (3) gives:

$$
\ln Y=\mathrm{g}(T)+\ln W
$$

Take $\mu$ equal to $\ln W$, specify the function $g$, and weather index $W$ can clearly be derived from a linear regression on:

$$
\ln Y=\mathrm{g}(T)+\mu
$$

where, by assumption, $\mu$ is a stochastic disturbance with expectation zero, a constant variance and no covariances between the elements of different years. The weather indexes can be derived from the estimated values of the disturbances $\mu$.

The empirical analysis uses a third degree orthogonal polynomial:

$$
\ln Y=\alpha_{0}+\alpha_{1} \cdot T+\alpha_{2} \cdot T^{2}+\alpha_{3} \cdot T^{3}+\mu
$$

where $T$ is a trend variable with an average value of zero during the total period. $T^{2}$ is the squared values of $T$, while $T^{3}$ has been transformed in such a way that the variable is orthogonal to $T$ and $T^{2}$ (Murdoch, 1966, p. 34). The $\alpha$ 's are the parameters of the systematic part.

Such a polynomial gives enough flexibility to allow differences in the systematic development during the period without adjusting excessively the estimated systematic part to extreme weather conditions. The parameters $\alpha_{1}, \alpha_{2}$ and $\alpha_{3}$ are tested by means of a Student $t$-test. When the $t$-value, relative to 0 , is smaller than 1.5 , for these parameters, the particular explanatory variable will be deleted. This is based on the prediction criterium used by Amemiya (1980, p. 334). The unconditional mean square prediction error forms the basis of this criterium.

As a result this method gives two output series:

1. The systematic or expected yield level for that product. This yield can be derived from (6) by setting $\mu=0$ and using the estimated $\alpha$ 's.

2. Weather indices for the particular product; this weather index is derived from:

$$
W=e^{\mu}=Y / \exp [\hat{\mathrm{g}}(T)]
$$

where $\hat{g}(T)$ is the estimated value of the polynomial trend function. 
$W$ can be rescaled so that the average index is 1.0 during the observation period; this implies 'average' weather during that period. However, rescaling prevents that actual yield levels can be 'revealed' exactly from expected yield and the weather index without using the rescaling factor.

\section{An additive model}

As an alternative for the multiplicative model in (2), an additive model can be used. The equivalent formulation of Equation (5) is:

$$
Y=\mathrm{h}(T)+\theta
$$

where $\mathrm{h}(T)$ is also an orthogonal polynomial trend function and $\theta$ is the disturbance of a linear regression equation with expectation zero and a constant variance. For an increasing systematic yield, such a model implies a smaller percent variation in yields. Here the systematic yield equals the estimated value of the polynomial trend $(\hat{h}(T))$, while the weather index is equal to $Y / \hat{h}(T)$.

Testing between these two different models can be done by means of testing the heteroscedasticity of the disturbances $\mu$ and $\theta$ for two subperiods (Judge et al., 1980, p. 131). The model with the smallest indication of heteroscedastic disturbances gives the best description of yield variation.

\section{Additional observations and the revision of weather indices}

The methodology introduced implies that a particular weather index should be revised over the complete observation period when new observations are available. The reason is that the trend function is estimated over the total period while also the average weather index over the total period is equal to 1.0. This can be rather cumbersome in practice. Therefore, a slightly different methodology will be used with constant weather indices over a historical period (here 1951-1985) while the weather index and the systematic yield for additional years can be revised with new observations. Due to the use of third degree polynomials yield changes at the end of an observation period can have large influences at the beginning of the observation period.

Empirical analyses show mostly that only indices for the first and last two or three years show some effect of increasing the length of the period. Therefore, we have estimated the trend function over the period 1948-1989 and we have gauged the average weather index at 1.0 over the period 1951-1985. Additional observations will only change the indices after 1985.

\section{Aggregation of weather indices}

The analysis for individual crops gives weather indices and normal yield levels for individual crops. We aren often interested in weather indices at a more aggregated level, such as for grains, potatoes or even for the whole arable sector. These weather 
indices will be constructed by means of weighing indices for particular products. Because the weather indices will be used for economic analyses, aggregation will be based on 'normal' value shares. Using the following definition:

$$
V_{i, t}=\sum_{j=-b}^{b} p_{i, t+j} \cdot q_{i, t+j}
$$

where: $V$ is the normalized value of a product (product $i$ in year $t$ ), $p$ is the price of a product, $q$ is the quantity of a product.

The value share for an individual product $i$ in period $t\left(s_{i, t}\right)$ is derived from:

$$
s_{i, t}=V_{i, t} /\left(\sum_{i=1}^{n} \mathrm{~V}_{\mathrm{i}, \mathrm{l}}\right)
$$

Here we assume a total of $n$ different products. These value shares have been used to derive aggregate weather indices. For some products (e.g. fodder crops), prices were not available. Here we assumed that an average hectare of fodder crops had the same revenue level as an average hectare of other crops.

Influences of prices and quantities in particular years will be smoothed by averaging over a period of five years $(b=2$ in Equation (9)). The five-year period has been shifted in the beginning and the end of the observation period.

\section{Results of the empirical analysis}

Table 1 gives the yield levels, which form the input for the empirical analysis.

The empirical analysis and the tests resulted in a set of normal yield functions are stated in Table 2, where one can find information on the type of furiction and the remaining parameters. Only two products (oats and pulse crops) show a complete third degree polynomial. These changes in systematic yield developments could be due to shifts in the areas of these products. The systematic share in the first and the second part of the period and the total period is also shown in Table 2. For eleven of the twelve products the systematic variance was more than 50 per cent of the total variance during the total period.

Table 3 shows the resulting weather indices for twelve products. All indices have an average value of 1.0 over the period 1948-1985. This implies the assumption of average weather over that particular period.

Table 4 shows aggregated weather indices for a number of products and the total arable sector. Two different indices for the arable sector have been calculated: the first excludes fodder crops (fodder beets and silage maize), while the second includes these products. Because an important share of the fodder crops is grown on mixed farms and specialized dairy farms the first index represents more the weather conditions at specialized arable farms. However, differences are small because weather indices are correlated (see also Table 6). Moreover, the share of fodder crops is always less than $17 \%$, although this share increased during the period 1948-1989. There are no observations for silage maize before 1954 . 
WEATHER INDICES FOR AGRICULTURAL PRODUCTION. 1

Table 1. Production (in $\mathrm{kg} \mathrm{ha}^{-1}$ ) of arable farm products. ${ }^{1}$

\begin{tabular}{|c|c|c|c|c|c|c|c|c|c|c|c|c|}
\hline \multirow[t]{2}{*}{ Year } & \multirow[t]{2}{*}{ Wheat } & \multirow[t]{2}{*}{ Barley } & \multirow[t]{2}{*}{ Oats } & \multirow[t]{2}{*}{ Rye } & \multicolumn{2}{|c|}{ Potatoes } & \multirow{2}{*}{ Sugar ${ }^{2}$} & \multirow{2}{*}{$\begin{array}{l}\text { Pulse } \\
\text { crops }\end{array}$} & \multirow{2}{*}{$\begin{array}{l}\text { Rape } \\
\text { seed }\end{array}$} & \multirow[t]{2}{*}{ Flax $^{3}$} & \multirow{2}{*}{$\begin{array}{l}\text { Fodder } \\
\text { beets }\end{array}$} & \multirow{2}{*}{$\begin{array}{l}\text { Silage } \\
\text { maize }^{4}\end{array}$} \\
\hline & & & & & ware & starch & & & & & & \\
\hline 1948 & 3230 & 2510 & 2230 & 2080 & 25900 & 27800 & 7249 & 1460 & 2100 & 8130 & 63900 & - \\
\hline 1949 & 4410 & 4000 & 3270 & 2830 & 24600 & 30500 & 6991 & 3020 & 2430 & 9090 & 58600 & - \\
\hline 1950 & 3309 & 3350 & 2710 & 2400 & 23600 & 26600 & 6916 & 2780 & 1400 & 7650 & 61500 & - \\
\hline 1951 & 3740 & 3110 & 3200 & 2850 & 23500 & 27100 & 5961 & 2750 & 1910 & 7390 & 51700 & - \\
\hline 1952 & 4059 & 3330 & 3160 & 2700 & 27100 & 27200 & 7276 & 3000 & 2390 & 8100 & 62400 & - \\
\hline 1953 & 3959 & 2610 & 3100 & 2510 & 24800 & 24000 & 7139 & 2520 & 2260 & 7340 & 65700 & - \\
\hline 1954 & 3870 & 3300 & 3270 & 3080 & 24400 & 25600 & 5959 & 2590 & 2710 & 7690 & 61100 & 8100 \\
\hline 1955 & 3859 & 3759 & 3409 & 3020 & 26900 & 28000 & 6973 & 3300 & 2530 & 8290 & 71000 & 10100 \\
\hline 1956 & 3800 & 3690 & 3160 & 2880 & 24500 & 22300 & 5725 & 2060 & 2510 & 7540 & 56600 & 7900 \\
\hline 1957 & 4240 & 4050 & 3180 & 2910 & 28200 & 28500 & 6630 & 3230 & 2530 & 7860 & 70200 & 9500 \\
\hline 1958 & 3959 & 3890 & 3240 & 2960 & 29000 & 24500 & 7568 & 3100 & 1970 & 7969 & 80600 & 10600 \\
\hline 1959 & 4620 & 3680 & 2550 & 2680 & 22000 & 26500 & 5794 & 3270 & 2900 & 7290 & 7800 & 10100 \\
\hline 1960 & 5010 & 4220 & 3390 & 3030 & 28500 & 31000 & 7928 & 3659 & 2700 & 8470 & 85000 & 9400 \\
\hline 1961 & 3950 & 3780 & 3509 & 2520 & 28500 & 29500 & 7189 & 3330 & 2510 & 8220 & 5900 & 9000 \\
\hline 1962 & 5030 & 4340 & 3900 & 3180 & 30500 & 32500 & 6307 & 3959 & 2500 & 8160 & 2100 & 7600 \\
\hline 1963 & 4480 & 3859 & 3780 & 2970 & 28500 & 30500 & 6513 & 3040 & 2520 & 8280 & 68900 & 8300 \\
\hline 1964 & 5010 & 4330 & 4090 & 3370 & 32500 & 34500 & 8232 & 3380 & 2840 & 8280 & 0400 & 9400 \\
\hline 1965 & 4550 & 3800 & 3620 & 2550 & 28500 & 23500 & 6635 & 2600 & 2590 & 7990 & 57800 & 8700 \\
\hline 1966 & 4280 & 3459 & 3600 & 2570 & 32500 & 31000 & 6440 & 2350 & 2600 & 7140 & 66000 & 9600 \\
\hline 1967 & 5100 & 4200 & 4100 & 3300 & 37500 & 35500 & 7878 & 3900 & 2900 & 8630 & 82500 & 11200 \\
\hline 1968 & 4700 & 3600 & 4200 & 3200 & 34000 & 35000 & 7375 & 3000 & 2700 & 8150 & 83000 & 9700 \\
\hline 1969 & 4700 & 3900 & 3900 & 3300 & 33000 & 31500 & 7808 & 3100 & 2000 & 7980 & 81000 & 9800 \\
\hline 1970 & & 3100 & 3700 & 3000 & & & 7234 & 3000 & 2900 & 6440 & 77500 & 11200 \\
\hline 1971 & 5200 & 3700 & 4600 & 3500 & 37500 & 37500 & 8232 & 3100 & 3150 & 8850 & 80000 & 10900 \\
\hline 1972 & 4400 & 4100 & 4200 & 2800 & 38000 & 37000 & 7128 & 2350 & 3000 & 9100 & 76500 & 8900 \\
\hline 1973 & 5400 & & & & & & 7267 & 2850 & & & & \\
\hline 1974 & 6000 & 4200 & 5000 & 3500 & 38500 & 40000 & 6930 & 3200 & 3250 & 8300 & 75500 & 10300 \\
\hline 1975 & 5100 & 4000 & 4600 & 3500 & 32900 & 33500 & 6742 & 3000 & 2600 & 6400 & 71500 & 12400 \\
\hline 1976 & 5700 & 4200 & 4100 & 3100 & 31000 & 28000 & 7161 & 2300 & 2800 & 6000 & 67500 & 8500 \\
\hline 1977 & 5400 & 4300 & 4500 & 3500 & 33700 & 35000 & 7347 & 3100 & 2750 & 7600 & 79000 & 11200 \\
\hline 1978 & 6800 & 4900 & 5600 & 4000 & 37300 & 40000 & 7759 & 3200 & 2450 & 8300 & 81000 & 12300 \\
\hline 1979 & 6100 & 4500 & 5200 & & 36000 & & 7253 & 3300 & 2650 & 7950 & & 12200 \\
\hline 1980 & 6400 & 4600 & 5200 & 4000 & 38000 & 33000 & 7742 & 2700 & 3600 & 7650 & 81000 & 12000 \\
\hline 1981 & 6900 & 4500 & 5400 & 3800 & 39900 & 38000 & 8478 & 3400 & 3400 & 8200 & 89000 & 13900 \\
\hline 1982 & 7600 & 5700 & 5700 & 4500 & 41100 & 32000 & 9341 & 4100 & 3100 & 7900 & 92500 & 13400 \\
\hline 1983 & 7100 & 4500 & 4500 & 3900 & 34100 & 31500 & 7207 & 3900 & 2800 & 6550 & 78500 & 12500 \\
\hline 1984 & 7900 & 5300 & 4800 & 4300 & 42700 & 40000 & 8153 & 4600 & 2850 & 9050 & 81500 & 10900 \\
\hline 1985 & 6700 & 5000 & 5200 & 4200 & 43200 & 41000 & 7808 & 3650 & 3050 & 8250 & 84500 & 11500 \\
\hline 1986 & 8100 & 6200 & 6100 & 4900 & 44400 & 35000 & 9576 & 5200 & 3450 & 7900 & 86500 & 13500 \\
\hline 1987 & 7000 & 5200 & 5200 & 4200 & 46000 & 43500 & 8532 & 3650 & 3200 & 8050 & 87500 & 11000 \\
\hline 1988 & 7400 & 4700 & 4600 & 4300 & 42400 & 41000 & 8938 & 3900 & 3300 & 7200 & 89000 & 13000 \\
\hline 1989 & 7700 & 4800 & 4100 & 5000 & 41500 & 41000 & 9672 & 4500 & 3650 & 6050 & 91500 & 13500 \\
\hline
\end{tabular}

1 All data have been used from LEI/CBS, Landbouwcijfers, several years. Data on sugar content are partly from Maandblad Suikerunie, several years.

2 Sugar beets have been calculated in kilogram sugar per hectare.

3 Flax has been based on both linseed and flax.

4 Yields of silage maize over the period $1954-1978$ are derived from te Velde $(1984$, p. 332$)$. Here a factor of 0.76 has been used to reduce the yields of RIVRO cultivars to actual yields of silage maize in the Netherlands. Silage maize is in kilogram of dry matter per hectare.

Netherlands Journal of Agricultural Science 39 (1991) 


\section{A. J. OSKAM}

Table 2. Estimation results of the systematic yield function; 1948-1989.

\begin{tabular}{|c|c|c|c|c|c|c|c|c|}
\hline \multirow[t]{3}{*}{ Product } & \multirow{3}{*}{$\begin{array}{l}\text { Type of } \\
\text { function }\end{array}$} & \multicolumn{3}{|c|}{ Parameters } & \multirow{3}{*}{$\begin{array}{l}\text { Average } \\
\text { share in } \\
\text { arable } \\
\text { production } \\
(\%)\end{array}$} & \multirow{2}{*}{\multicolumn{3}{|c|}{$\begin{array}{l}\text { Share of systematic } \\
\text { variance in total variance }\end{array}$}} \\
\hline & & \multirow{2}{*}{$\alpha_{1}$} & \multirow{2}{*}{$\alpha_{2}$} & \multirow{2}{*}{$\alpha_{3}$} & & & & \\
\hline & & & & & & $\begin{array}{l}1948- \\
1968\end{array}$ & $\begin{array}{l}1968- \\
1989\end{array}$ & $\begin{array}{l}\text { total } \\
\text { period }\end{array}$ \\
\hline Wheat & $\log$ & $\begin{array}{l}0.019 \\
(0.001)^{1}\end{array}$ & $\begin{array}{l}0.00021 \\
(0.00010)\end{array}$ & - & 14.1 & 0.503 & 0.687 & 0.890 \\
\hline Barley & lin & $\begin{array}{l}48.5 \\
(5.8)\end{array}$ & - & - & 6.9 & 0.360 & 0.188 & 0.631 \\
\hline Oats & $\log$ & $\begin{array}{l}0.016 \\
(0.001)\end{array}$ & $\begin{array}{l}-0.00025 \\
(0.00012)\end{array}$ & $\begin{array}{l}-0.000024 \\
(0.000012)\end{array}$ & 6.9 & 0.629 & 0.193 & 0.782 \\
\hline Rye & lin & $\begin{array}{l}49.6 \\
(3.7)\end{array}$ & $\begin{array}{l}1.26 \\
(0.34)\end{array}$ & - & 5.9 & 0.215 & 0.645 & 0.830 \\
\hline Potatoes: & & & & & & & & \\
\hline - ware & $\log$ & $\begin{array}{l}0.015 \\
(0.001)\end{array}$ & - & - & 25.7 & 0.446 & 0.744 & 0.856 \\
\hline - starch & $\log$ & $\begin{array}{l}0.011 \\
(0.001)\end{array}$ & - & - & 8.2 & 0.278 & 0.381 & 0.623 \\
\hline Sugar beets & lin & $\begin{array}{l}51.7 \\
(8.4)\end{array}$ & $\begin{array}{l}2.1 \\
(0.8)\end{array}$ & - & 8.2 & 0.015 & 0.457 & 0.533 \\
\hline Pulse crops & $\operatorname{lin}$ & $\begin{array}{l}32.9 \\
(6.5)\end{array}$ & $\begin{array}{l}1.0 \\
(0.6)\end{array}$ & $\begin{array}{l}0.19 \\
(0.06)\end{array}$ & 2.9 & 0.232 & 0.540 & 0.511 \\
\hline Rape seed & $\log$ & $\begin{array}{l}0.011 \\
(0.002)\end{array}$ & - & $\begin{array}{l}0.000023 \\
(0.000014)\end{array}$ & 0.8 & 0.338 & 0.465 & 0.632 \\
\hline Flax & - & - & - & - & 2.2 & 0 & 0 & 0 \\
\hline Fodder beets & lin & $\begin{array}{l}709 \\
(104)\end{array}$ & - & - & 2.4 & 0.140 & 0.460 & 0.536 \\
\hline Silage maize & $\log$ & $\begin{array}{l}0.012 \\
(0.002)\end{array}$ & - & - & 5.7 & 0.232 & 0.311 & 0.573 \\
\hline
\end{tabular}

1 Estimated standard deviations between parentheses. 
Table 3. Basic weather indices of Dutch arable farm products.

\begin{tabular}{|c|c|c|c|c|c|c|c|c|c|c|c|c|}
\hline \multirow[t]{2}{*}{ Year } & \multirow[t]{2}{*}{ Wheat } & \multirow[t]{2}{*}{ Barley } & \multirow[t]{2}{*}{ Oats } & \multirow[t]{2}{*}{ Rye } & \multicolumn{2}{|c|}{ Potatoes } & \multirow{2}{*}{$\begin{array}{l}\text { Sugar } \\
\text { beets }\end{array}$} & \multirow{2}{*}{$\begin{array}{l}\text { Pulse } \\
\text { crops }\end{array}$} & \multirow{2}{*}{$\begin{array}{l}\text { Rape } \\
\text { seed }\end{array}$} & \multirow[t]{2}{*}{ Flax } & \multirow{2}{*}{$\begin{array}{l}\text { Fodder } \\
\text { beets }\end{array}$} & \multirow{2}{*}{$\begin{array}{l}\text { Silage } \\
\text { maize }\end{array}$} \\
\hline & & & & & ware & starch & & & & & & \\
\hline 1948 & 0.883 & 0.806 & 0.780 & 0.780 & 1.089 & 1.085 & 1.049 & 0.665 & 1.055 & 1.001 & 1.075 & - \\
\hline 1949 & 1.192 & 1.266 & 1.139 & 1.061 & 1.019 & 1.178 & 1.016 & 1.277 & 1.181 & 1.130 & 0.974 & - \\
\hline 1950 & 0.885 & 1.044 & 0.938 & 0.899 & 0.963 & 1.016 & 1.009 & 1.106 & 0.660 & 0.951 & 1.010 & - \\
\hline 1951 & 0.981 & 0.962 & 1.089 & 1.059 & 0.944 & 1.025 & 0.878 & 1.041 & 0.866 & 0.924 & 0.842 & - \\
\hline 1952 & 1.052 & 1.015 & 1.064 & 1.001 & 1.072 & 1.017 & 1.075 & 1.089 & 1.057 & 1.018 & 1.005 & - \\
\hline 1953 & 1.014 & 0.784 & 1.031 & 0.927 & 0.966 & 0.888 & 1.057 & 0.884 & 0.977 & 0.924 & 1.046 & - \\
\hline 1954 & 0.978 & 0.977 & 1.072 & 1.133 & 0.936 & 0.936 & 0.884 & 0.884 & 1.148 & 0.971 & 0.962 & 0.950 \\
\hline 1955 & 0.963 & 1.098 & 1.100 & 1.105 & 1.017 & 1.013 & 1.035 & 1.101 & 1.052 & 1.050 & 1.106 & 1.170 \\
\hline 1956 & 0.935 & 1.062 & 1.002 & 1.047 & 0.912 & 0.798 & 0.850 & 0.676 & 1.027 & 0.955 & 0.872 & 0.904 \\
\hline 1957 & 1.029 & 1.150 & 0.990 & 1.051 & 1.034 & 1.008 & 0.984 & 1.045 & 1.020 & 0.997 & 1.070 & 1.075 \\
\hline 1958 & 0.947 & 1.090 & 0.989 & 1.060 & 1.047 & 0.857 & 1.123 & 0.994 & 0.784 & 1.012 & 1.215 & 1.185 \\
\hline 1959 & 1.089 & 1.017 & 0.762 & 0.952 & 0.783 & 0.917 & 0.858 & 1.042 & 1.141 & 0.925 & 0.564 & 1.116 \\
\hline 1960 & 1.162 & 1.151 & 0.992 & 1.066 & 0.999 & 1.061 & 1.172 & 1.162 & 1.051 & 1.076 & 1.255 & 1.026 \\
\hline 1961 & 0.902 & 1.018 & 1.004 & 0.877 & 0.984 & 0.998 & 1.060 & 1.056 & 0.968 & 1.043 & 1.109 & 0.971 \\
\hline 1962 & 1.130 & 1.153 & 1.091 & 1.095 & 1.037 & 1.087 & 0.927 & 1.257 & 0.957 & 1.035 & 0.898 & 0.810 \\
\hline 1963 & 0.990 & 1.013 & 1.033 & 1.010 & 0.954 & 1.009 & 0.953 & 0.967 & 0.958 & 1.050 & 0.986 & 0.874 \\
\hline 1964 & 1.089 & 1.122 & 1.092 & 1.132 & 1.072 & 1.129 & 1.199 & 1.080 & 1.073 & 1.049 & 1.139 & 0.978 \\
\hline 1965 & 0.972 & 0.973 & 0.944 & 0.845 & 0.926 & 0.760 & 0.962 & 0.835 & 0.973 & 1.011 & 0.811 & 0.895 \\
\hline 1966 & 0.898 & 0.875 & 0.917 & 0.840 & 1.040 & 0.992 & 0.928 & 0.759 & 0.971 & 0.902 & 0.917 & 0.976 \\
\hline 1967 & 1.050 & 1.049 & 1.020 & 1.063 & 1.182 & 1.123 & 1.129 & 1.266 & 1.078 & 1.091 & 1.135 & 1.125 \\
\hline 1968 & 0.950 & 0.889 & 1.021 & 1.015 & 1.056 & 1.095 & 1.049 & 0.980 & 0.999 & 1.029 & 1.131 & 0.963 \\
\hline 1969 & 0.932 & 0.951 & 0.927 & 1.031 & 1.009 & 0.975 & 1.103 & 1.018 & 0.737 & 1.006 & 1.093 & 0.961 \\
\hline 1970 & 0.953 & 0.747 & 0.860 & 0.922 & 1.070 & 1.086 & 1.014 & 0.990 & 1.063 & 0.809 & 1.036 & 1.085 \\
\hline 1971 & 0.991 & 0.882 & 1.047 & 1.058 & 1.113 & 1.135 & 1.144 & 1.026 & 1.149 & 1.117 & 1.059 & 1.044 \\
\hline 1972 & 0.821 & 0.966 & 0.936 & 0.832 & 1.111 & 1.107 & 0.982 & 0.780 & 1.089 & 1.149 & 1.004 & 0.842 \\
\hline 1973 & 0.987 & 0.979 & 0.962 & 0.992 & 1.060 & 1.095 & 0.992 & 0.945 & 0.956 & 1.034 & 0.975 & 0.935 \\
\hline 1974 & 1.074 & 0.968 & 1.073 & 1.003 & 1.092 & 1.171 & 0.937 & 1.059 & 1.166 & 1.048 & 0.972 & 0.952 \\
\hline 1975 & 0.893 & 0.911 & 0.971 & 0.985 & 0.919 & 0.969 & 0.902 & 0.988 & 0.926 & 0.806 & 0.913 & 1.132 \\
\hline 1976 & 0.976 & 0.947 & 0.852 & 0.856 & 0.853 & 0.801 & 0.948 & 0.751 & 0.989 & 0.757 & 0.854 & 0.767 \\
\hline 1977 & 0.904 & 0.959 & 0.922 & 0.948 & 0.913 & 0.990 & 0.962 & 1.001 & 0.963 & 0.963 & 0.990 & 0.999 \\
\hline 1978 & 1.113 & 1.081 & 1.133 & 1.063 & 0.996 & 1.119 & 1.004 & 1.018 & 0.849 & 1.055 & 1.007 & 1.084 \\
\hline 1979 & 0.975 & 0.982 & 1.041 & 1.042 & 0.947 & 1.107 & 0.928 & 1.030 & 0.908 & 1.014 & 0.973 & 1.062 \\
\hline 1980 & 0.999 & 0.994 & 1.032 & 1.021 & 0.984 & 0.903 & 0.978 & 0.824 & 1.217 & 0.980 & 0.989 & 1.032 \\
\hline 1981 & 1.051 & 0.962 & 1.065 & 0.951 & 1.018 & 1.028 & 1.057 & 1.011 & 1.133 & 1.055 & 1.078 & 1.182 \\
\hline 1982 & 1.130 & 1.206 & 1.120 & 1.103 & 1.033 & 0.856 & 1.150 & 1182 & 1.016 & 1.022 & 1.111 & 1.126 \\
\hline 1983 & 1.029 & 0.943 & 0.883 & 0.936 & 0.844 & 0.834 & 0.876 & 1.087 & 0.901 & 0.856 & 0.934 & 1.038 \\
\hline 1984 & 1.116 & 1.099 & 0.942 & 1.011 & 1.041 & 1.047 & 0.977 & 1.234 & 0.899 & 1.182 & 0.962 & 0.894 \\
\hline 1985 & 0.922 & 1.027 & 1.025 & 0.967 & 1.037 & 1.061 & 0.923 & 0.939 & 0.940 & 1.088 & 0.989 & 0.932 \\
\hline 1986 & 1.094 & 1.251 & 1.220 & 1.112 & 1.051 & 0.894 & 1.108 & 1.278 & 1.050 & 1.051 & 1.001 & 1.085 \\
\hline 1987 & 0.921 & 1.039 & 1.050 & 0.933 & 1.073 & 1.099 & 0.973 & 0.855 & 0.948 & 1.080 & 1.004 & 0.873 \\
\hline 1988 & 0.947 & 0.930 & 0.941 & 0.935 & 0.974 & 1.024 & 1.005 & 0.868 & 0.949 & 0.983 & 1.013 & 1.020 \\
\hline 1989 & 0.959 & 0.941 & 0.852 & 1.064 & 0.939 & 1.013 & 1.071 & 0.949 & 1.016 & 0.849 & 1.034 & 1.047 \\
\hline
\end{tabular}




\section{A. J. OSKAM}

Table 4. Weather indices of aggregate products in Dutch arable farming. ${ }^{1}$

\begin{tabular}{|c|c|c|c|c|c|}
\hline Year & Cereals & Potatoes & Fodder crops & $\begin{array}{l}\text { Total excluding } \\
\text { fodder crops }\end{array}$ & $\begin{array}{l}\text { Total including } \\
\text { fodder crops }\end{array}$ \\
\hline 1948 & 0.807 & 1.088 & 1.075 & 0.939 & 0.947 \\
\hline 1949 & 1.143 & 1.056 & 0.974 & 1.107 & 1.099 \\
\hline 1950 & 0.931 & 0.975 & 1.010 & 0.959 & 0.962 \\
\hline 1951 & 1.037 & 0.962 & 0.842 & 0.989 & 0.980 \\
\hline 1952 & 1.033 & 1.060 & 1.005 & 1.048 & 1.045 \\
\hline 1953 & 0.955 & 0.949 & 1.046 & 0.958 & 0.964 \\
\hline 1954 & 1.053 & 0.936 & 0.962 & 0.987 & 0.985 \\
\hline 1955 & 1.069 & 1.016 & 1.106 & 1.051 & 1.054 \\
\hline 1956 & 1.007 & 0.887 & 0.872 & 0.932 & 0.929 \\
\hline 1957 & 1.045 & 1.028 & 1.070 & 1.030 & 1.032 \\
\hline 1958 & 1.012 & 1.001 & 1.215 & 1.024 & 1.034 \\
\hline 1959 & 0.964 & 0.817 & 0.579 & 0.909 & 0.894 \\
\hline 1960 & 1.101 & 1.014 & 1.247 & 1.091 & 1.098 \\
\hline 1961 & 0.945 & 0.987 & 1.103 & 0.983 & 0.988 \\
\hline 1962 & 1.120 & 1.051 & 0.893 & 1.071 & 1.064 \\
\hline 1963 & 1.008 & 0.968 & 0.977 & 0.988 & 0.988 \\
\hline 1964 & 1.103 & 1.086 & 1.121 & 1.112 & 1.112 \\
\hline 1965 & 0.948 & 0.885 & 0.824 & 0.931 & 0.928 \\
\hline 1966 & 0.888 & 1.027 & 0.929 & 0.938 & 0.937 \\
\hline 1967 & 1.046 & 1.167 & 1.133 & 1.109 & 1.110 \\
\hline 1968 & 0.956 & 1.066 & 1.084 & 1.017 & 1.019 \\
\hline 1969 & 0.948 & 0.999 & 1.043 & 1.001 & 1.002 \\
\hline 1970 & 0.886 & 1.074 & 1.065 & 0.991 & 0.993 \\
\hline 1971 & 0.979 & 1.119 & 1.050 & 1.078 & 1.076 \\
\hline 1972 & 0.872 & 1.110 & 0.871 & 1.001 & 0.995 \\
\hline 1973 & 0.983 & 1.070 & 0.942 & 1.021 & 1.016 \\
\hline 1974 & 1.041 & 1.110 & 0.956 & 1.051 & 1.043 \\
\hline 1975 & 0.912 & 0.930 & 1.126 & 0.919 & 0.939 \\
\hline 1976 & 0.949 & 0.842 & 0.772 & 0.894 & 0.880 \\
\hline 1977 & 0.922 & 0.930 & 1.001 & 0.937 & 0.944 \\
\hline 1978 & 1.105 & 1.023 & 1.085 & 1.037 & 1.043 \\
\hline 1979 & 0.985 & 0.989 & 1.064 & 0.971 & 0.983 \\
\hline 1980 & 1.001 & 0.962 & 1.035 & 0.978 & 0.985 \\
\hline 1981 & 1.033 & 1.020 & 1.184 & 1.035 & 1.056 \\
\hline 1982 & 1.142 & 0.995 & 1.129 & 1.073 & 1.081 \\
\hline 1983 & 1.004 & 0.842 & 1.039 & 0.895 & 0.917 \\
\hline 1984 & 1.103 & 1.042 & 0.898 & 1.044 & 1.020 \\
\hline 1985 & 0.946 & 1.043 & 0.936 & 0.985 & 0.977 \\
\hline 1986 & 1.131 & 1.013 & 1.084 & 1.076 & 1.077 \\
\hline 1987 & 0.951 & 1.079 & 0.875 & 1.010 & 0.988 \\
\hline 1988 & 0.943 & 0.985 & 1.020 & 0.977 & 0.984 \\
\hline 1989 & 0.953 & 0.955 & 1.046 & 0.988 & 0.998 \\
\hline Average & & & & 1.003 & 1.004 \\
\hline
\end{tabular}

I Annual value shares and centered value shares are available on request from the author. 
Table 5. Normalized production in $\mathrm{kg}$ per hectare of arable farm products.

\begin{tabular}{|c|c|c|c|c|c|c|c|c|c|c|c|c|}
\hline \multirow[t]{2}{*}{ Year } & \multirow[t]{2}{*}{ Wheat } & \multirow[t]{2}{*}{ Barley } & \multirow[t]{2}{*}{ Oats } & \multirow[t]{2}{*}{ Rye } & \multicolumn{2}{|c|}{ Potatoes } & \multirow[t]{2}{*}{ Sugar } & \multirow{2}{*}{$\begin{array}{l}\text { Pulse } \\
\text { crops }\end{array}$} & \multirow{2}{*}{$\begin{array}{l}\text { Rape } \\
\text { seed }\end{array}$} & \multirow[t]{2}{*}{ Flax } & \multirow{2}{*}{$\begin{array}{l}\text { Fodder } \\
\text { beets }\end{array}$} & \multirow{2}{*}{$\begin{array}{l}\text { Silage } \\
\text { maize }\end{array}$} \\
\hline & & & & & ware & starch & & & & & & \\
\hline 1948 & 3659 & 3112 & 2857 & 2667 & 23783 & $25614 \mathrm{v}$ & 6912 & 2195 & 1990 & 7847 & 59459 & - \\
\hline 1949 & 3698 & 3160 & 2870 & 2667 & 24145 & 25902 & 6881 & 2364 & 2057 & 7847 & 60168 & - \\
\hline 1950 & 3739 & 3209 & 2889 & 2668 & 24513 & 26192 & 6854 & 2513 & 2119 & 7847 & 60877 & - \\
\hline 1951 & 3783 & 3257 & 2915 & 2673 & 24886 & 26486 & 6831 & 2643 & 2178 & 7847 & 61586 & - \\
\hline 1952 & 3828 & 3306 & 2946 & 2680 & 25265 & 26784 & 6812 & 2756 & 2233 & 7847 & 62295 & - \\
\hline 1953 & 3875 & 3354 & 2983 & 2689 & 25650 & 27084 & 6797 & 2852 & 2284 & 7847 & 63004 & - \\
\hline 1954 & 3925 & 3403 & 3026 & 2701 & 26041 & 27388 & 6786 & 2932 & 2331 & 7847 & 63713 & 8505 \\
\hline 1955 & 3977 & 3451 & 3075 & 2715 & 26438 & 27696 & 6780 & 2998 & 2374 & 7847 & 64422 & 8606 \\
\hline 1956 & 4032 & 3500 & 3128 & 2732 & 26841 & 28006 & 6778 & 3051 & 2413 & 7847 & 65131 & 8710 \\
\hline 1957 & 4089 & 3548 & 3187 & 2751 & 27249 & 28321 & 6779 & 3092 & 2449 & 7847 & 65839 & 8814 \\
\hline 1958 & 4149 & 3597 & 3250 & 2773 & 27665 & 28639 & 6785 & 3122 & 2481 & 7847 & 66548 & 8919 \\
\hline 1959 & 4211 & 3645 & 3318 & 2798 & 28086 & 28960 & 6795 & 3141 & 2510 & 7847 & 67257 & 9026 \\
\hline 1960 & 4276 & 3694 & 3390 & 2825 & 28514 & 29285 & 6810 & 3153 & 2536 & 7847 & 67966 & 9134 \\
\hline 1961 & 4344 & 3742 & 3466 & 2854 & 28948 & 29614 & 6828 & 3156 & 2559 & 7847 & 68675 & 9244 \\
\hline 1962 & 4415 & 3791 & 3546 & 2886 & 29389 & 29946 & 6851 & 3153 & 2580 & 7847 & 69384 & 9354 \\
\hline 1963 & 4489 & 3839 & 3629 & 2920 & 29837 & 30383 & 6878 & 3145 & 2598 & 7847 & 70093 & 9466 \\
\hline 1964 & 4567 & 3888 & 3716 & 2957 & 30292 & 30622 & 6908 & 3133 & 2614 & 7847 & 70802 & 9580 \\
\hline 1965 & 4647 & 3936 & 3804 & 2997 & 30753 & 30966 & 6944 & 3117 & 2629 & 7847 & 71511 & 9694 \\
\hline 1966 & 4731 & 3985 & 3895 & 3039 & 31222 & 31314 & 6983 & 3100 & 2643 & 7847 & 72220 & 9810 \\
\hline 1967 & 4819 & 4033 & 3987 & 3083 & 31697 & 31665 & 7026 & 3081 & 2656 & 7847 & 72929 & 9928 \\
\hline 1968 & 4910 & 4082 & 4081 & 3130 & 32180 & 32020 & 7074 & 3063 & 2668 & 7847 & 73638 & 10047 \\
\hline 1969 & 5005 & 4130 & 4174 & 3180 & 32670 & 32380 & 7125 & 3047 & 2680 & 7847 & 74347 & 10167 \\
\hline 1970 & 5104 & 4179 & 4267 & 3232 & 33168 & 32743 & 7181 & 3033 & 2693 & 7847 & 75056 & 10289 \\
\hline 1971 & 5207 & 4227 & 4360 & 3287 & 33673 & 33111 & 7241 & 3022 & 2706 & 7847 & 75765 & 10412 \\
\hline 1972 & 5315 & 4276 & 4450 & 3344 & 34186 & 33482 & 7305 & 3017 & 2720 & 7847 & 76474 & 10536 \\
\hline 1973 & 5427 & 4324 & 4538 & 3403 & 34707 & 33858 & 7374 & 3017 & 2735 & 7847 & 77183 & 10663 \\
\hline 1974 & 5544 & 4373 & 4622 & 3466 & 35236 & 34238 & 7446 & 3024 & 2753 & 7847 & 77892 & 10790 \\
\hline 1975 & 5666 & 4421 & 4701 & 3530 & 35773 & 34623 & 7523 & 3039 & 2772 & 7847 & 78601 & 10919 \\
\hline 1976 & 5793 & 4470 & 4775 & 3597 & 36318 & 35011 & 7604 & 3064 & 2795 & 7847 & 79309 & 11050 \\
\hline 1977 & 5925 & 4518 & 4843 & 3667 & 36871 & 35404 & 7689 & 3099 & 2820 & 7847 & 80018 & 11182 \\
\hline 1978 & 6063 & 4567 & 4903 & 3739 & 37433 & $35801 \mathrm{v}$ & 7778 & 3146 & 2849 & 7847 & 80727 & 11316 \\
\hline 1979 & 6206 & 4615 & 4955 & 3814 & 38003 & 36203 & 7871 & 3205 & 2882 & 7847 & 81436 & 11452 \\
\hline 1980 & 6356 & 4664 & 4997 & 3891 & 38582 & 36610 & 7968 & 3278 & 2920 & 7847 & 82145 & 11589 \\
\hline 1981 & 6513 & 4712 & 5028 & 3971 & 39170 & 37021 & 8070 & 3366 & 2963 & 7847 & 82854 & 11728 \\
\hline 1982 & 6676 & 4761 & 5049 & 4053 & 39766 & 37436 & 8176 & 3470 & 3013 & 7847 & 83563 & 11868 \\
\hline 1983 & 6846 & 4809 & 5057 & 4138 & 40372 & 37856 & 8285 & 3592 & 3068 & 7847 & 84272 & 12010 \\
\hline 1984 & 7023 & 4858 & 5052 & 4225 & 40987 & 38281 & 8399 & 3731 & 3132 & 7847 & 84981 & 12154 \\
\hline 1985 & 7208 & 4906 & 5033 & 4315 & 41612 & 38711 & 8518 & 3890 & 3203 & 7847 & 85690 & 12300 \\
\hline 1986 & 7401 & 4955 & 5000 & 4408 & 42246 & 39145 & 8640 & 4069 & 3284 & 7847 & 86399 & 12447 \\
\hline 1987 & 7602 & 5003 & 4952 & 4502 & 42889 & 39585 & 8766 & 4270 & 3375 & 7847 & 87108 & 12596 \\
\hline 1988 & 7812 & 5052 & 4890 & 4600 & 43543 & 40029 & 8897 & 4494 & 3477 & 7847 & 87817 & 12747 \\
\hline 1989 & 8032 & 5100 & 4812 & 4700 & 44206 & 40478 & 9032 & 4742 & 3593 & 7847 & 88526 & 12899 \\
\hline $\begin{array}{l}\text { Average } \\
\text { growth } \\
\left(\% \text { year }{ }^{-1}\right)\end{array}$ & 1.94 & 1.21 & 1.28 & 1.39 & 1.52 & 1.12 & 0.65 & 1.90 & 1.45 & 0 & 0.98 & 1.20 \\
\hline
\end{tabular}




\section{A. J. OSKAM}

The normalized production levels are in Table 5. Most products show a positive trend in the normalized production per hectare. Flax is an exception: no significant trend could be detected, and a constant normal yield level was the result. This can be due to the large changes in area with different potential yields. There are remarkable differences in systematic yield developments between the different products. Figure 1 gives an overview of the weighted annual increase of these yields. Here we observe first a slightly declining and later on an increasing development in the growth of yields. Average annual yields increased by 1.17 and $1.48 \%$ over the periods 1949-1969 and 1969-1988, respectively. The figure illustrates the continuous, development in yields during a longer period. Annual increases are now at a level of nearly $1.7 \%$.

Comparing systematic yield developments in the Netherlands and the UK, annual increases are about $30 \%$ lower in the Netherlands (Britton, 1990, p. 5). Annual increases of wheat yield in the Netherlands are about $20 \%$ lower than that of the 'Total world excluding China' (Anderson \& Hazell, 1989, p. 24). This illustrates that long-term growth rates are relatively low in the Netherlands. A number of related measures has been presented in Tables 6 and 7 .

Table 6 gives correlations between all weather indices of individual products and the aggregated index. They illustrate that all products have a positive correlation with the aggregate weather index. The correlation for ware potatoes and sugar beets are highest, also due to the large share of these products in total arable production. A principal component analysis on 11 'individual' weather indices indicated that the

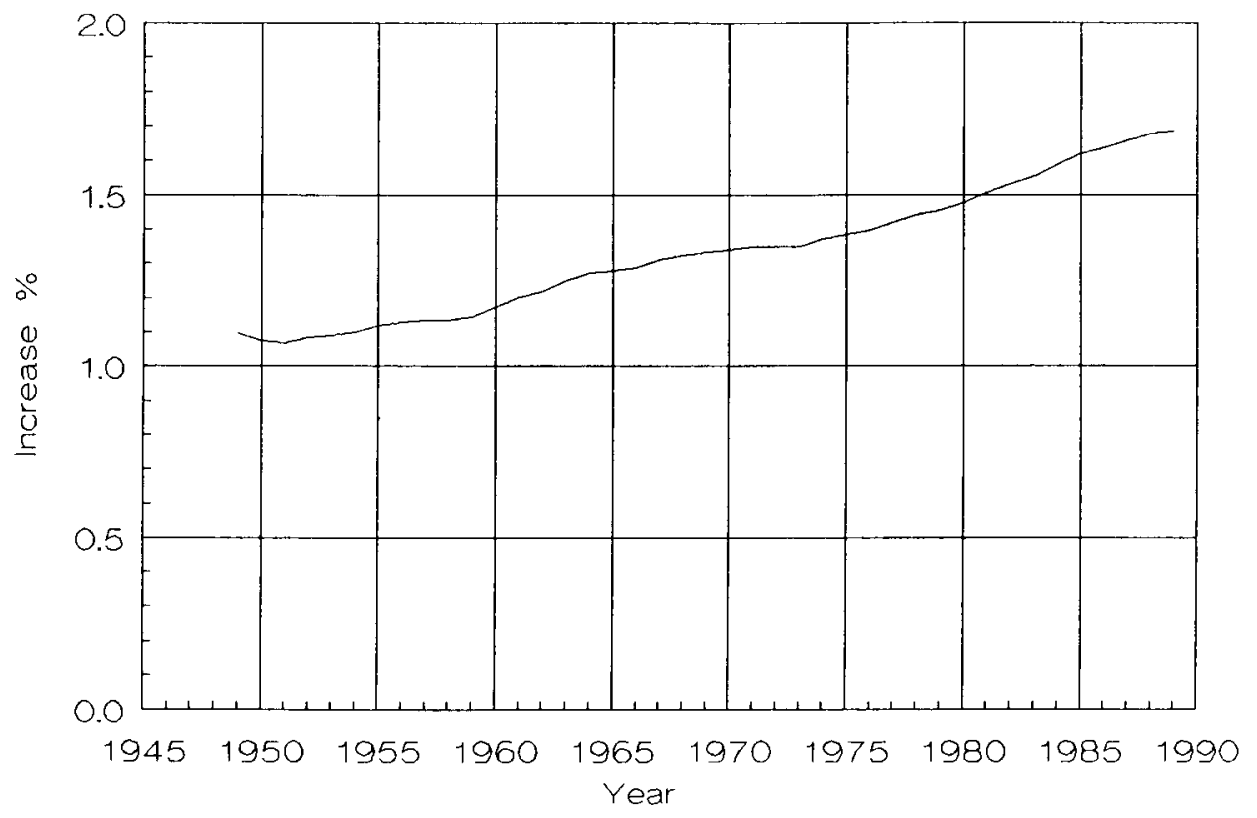

Fig. 1. Weighted annual yield increase of arable crops (1949-1989); systematic increase. 
first principal component represented $40 \%$ of the total variance, the first three principal components took $70 \%$ and the first five components $85 \%$. This implies that weather cannot be represented by one particular factor alone (a linear combination of individual weather indices) or by one representative crop. The value share of a particular product is, of course, an important indicator to incorporate a specific weather index. However, for similar products (like potatoes or cereals) the main product alone may be sufficient in calculating an aggregated weather index.

Table 6. Correlation between weather indices of particular crops and the weather index of arable products.

\begin{tabular}{ll}
\hline Product & Correlation \\
Wheat & 0.55 \\
Barley & 0.44 \\
Oats & 0.71 \\
Rye & 0.65 \\
Ware potatoes & 0.74 \\
Starch potatoes & 0.38 \\
Sugar beets & 0.73 \\
Pulse crops & 0.55 \\
Rape seed & 0.31 \\
Flay & 0.64 \\
Fodder beets & 0.72 \\
Silage maize & 0.32 \\
\hline
\end{tabular}

1 Period 1954-1989.

Table 7. Standard deviations of weather indices during two different subperiods.

\begin{tabular}{lll}
\hline Product & \multicolumn{2}{l}{ Period } \\
\cline { 2 - 3 } & $1948-1968$ & $1969-1989$ \\
Wheat & 0.090 & 0.083 \\
Barley & 0.119 & 0.107 \\
Oats & 0.096 & 0.100 \\
Rye & 0.103 & 0.074 \\
Ware potatoes & 0.082 & 0.078 \\
Starch potatoes & 0.108 & 0.106 \\
Sugar beets & 0.101 & 0.078 \\
Pulse crops & 0.175 & 0.135 \\
Rape seed & 0.119 & 0.116 \\
Flax & 0.061 & 0.117 \\
Fodder beets & 0.156 & 0.059 \\
Silage maize & 0.1011 & 0.107 \\
Total excluding fodder crops & 0.065 & 0.055 \\
Total including fodder crops & 0.066 & 0.053 \\
\hline
\end{tabular}

1 Period 1954-1968. 


\section{A. J. OSKAM}

\section{Discussion}

As expected, weather indices for individual crops vary more than weather indices for the total arable sector (see Table 7). Only with a perfect positive correlation between the weather indices for the individual products, would the resulting index be a weighted average of individual indices. The most extreme observed difference in indices is about $23 \%$ of a normal harvest (between the years 1964 and 1976) as can be observed in Table 4. Furthermore, calculations over the period 1948-1989 indicate that $30 \%$ of the total variance of 'weighted yields' was due to weather variation and $70 \%$ to systematic variation. Increasing the period implies that the share of weather variation is declining. The systematic developments in yield accounted for 33 and $53 \%$ of total variation in 1948-1968 and 1969-1989, respectively. This difference is partly due to the smaller increase of yields in the first period (see Figure $1)$. For an average year the systematic development is about $1.3 \%$ of a normal yield, while the weather variation is nearly $6 \%$; a good illustration that weather variation is important in short-term periods.

A second point of investigation is the variation, due to changing weather in the first and the second half of the total period. First of all we tested if the weather index was significantly different from a normal variable with expectation 1 . With a $5 \%$ level of significance we could not reject the hypothesis that the weather index had a normal distribution. Using a Komolgorov-Smirnov test, no difference could be found between the weather index and a normal variable. All individual crops showed no significant differences with the normal distribution, even at the $20 \%$ significance level. Some crops had a slight positive skewness, others a small (but insignificant) negative.

The standard deviation of yield, in relation to 'normal' yields is represented by the standard deviations of the weather indices. They have been shown in Table 7 . Relative yield variability is mostly lower in the second part of the period, although there are differences between crops, while at an aggregate level yield variability is reduced by $15-20 \%$. Yield levels, however, increase by more than $20 \%$ in the second part of the period compared with the first part.

Comparisons of our results with the work of Stallings $(1960,1961)$ show that variation due to weather conditions is much smaller in the Netherlands than in the United States. Other research sources on yield variation confirm this conclusion (Anderson \& Hazell, 1989; Weber \& Sievers, 1985). However, relative yield variations of cereals in the Netherlands are comparable to variations in countries such as France, Germany and the UK.

\section{Critical remarks}

A number of remarks can be made about the methodology applied. Most important is that all stochastic factors influencing the yields of arable products are considered as weather effects; also diseases and plagues are contained in the weather indices. This implies that non-smooth changes in economic conditions (e.g. price changes of products or inputs influencing the optimum yield level) are also incorporated in the 
weather effects. The effects of these biases are unimportant, however, when yield levels show large variation due to weather conditions and small variations due to price variation. Due to relative small variation of expected prices and also small variation of the optimum yield levels (de Wit, 1988), these biases seem to be unimportant.

Another point is that some variation in production will now show up in yield levels. Also the area of a particular crop can be influenced by weather conditions. To give an example, a part of the winter wheat can be substituted by spring barley when the winter weather is severe. This may pass unobserved because both winter wheat and spring barley may show normal yield levels. We assume, however, that in the Netherlands differences between planned and actual areas for the particular crops are small due to limited weather variation in winter and spring.

Systematic yield levels can be influenced by technical developments as well as by important changes in the area of products. Important shifts in the area of product can have a large influence on the average expected yield. Especially green maize, cole seed, flax, fodder beets, oats and rye show important area shifts.

\section{Concluding marks}

Although the calculated weather indices and the related normalized yields have a number of drawbacks, they form the only consistent long-term source in this area. Therefore, these numbers could be useful for several types of economic research. The numbers for individual products can also be useful for technical research. They give a comprehensive overview over a longer period.

Here we have calculated numbers for the Netherlands. The same methodology can be used for regional data. Weather indices for individual crops could be used for the calculation of weather indices at an individual farm level. Such an index depends on the composition of crops produced. It might be, however, that regional differences between farms are also important.

\section{References}

Amemiya, T., 1980. Selection of regressors. International Economic Review 21: 321-354.

Anderson, J. R.\& P. B. R. Hazell (Eds), 1989. Variability in grain yields. The Johns Hopkins University Press, Baltimore, $395 \mathrm{pp}$.

Britton, D. (Ed.), 1990. Agriculture in Britain: changing pressures and policies. Commonwealth Agricultural Bureau International, Wallingford, $215 \mathrm{pp}$.

Chambers, R. G., 1988. Applied production analysis; A dual approach. Cambridge University Press, Cambridge, $331 \mathrm{pp}$.

Cromarty, W. A., 1959. An econometric model for United States Agriculture. Journal of the American Statistical Association XXX: 556-574.

Doll, J. P., 1967. An analytical technique for estimating weather indices from meteorological measurements. Journal of Farm Economics 49: 79-88.

Judge, G. C., W. E. Griffiths, R. C. Hill \& T-C. Lee, 1980. The theory and practice of econometrics. Wiley, New York, $810 \mathrm{pp}$.

Murdoch, D. C., 1966. Linear Algebra for Undergraduates. Wiley, New York, 239 pp.

Netherlands Journal of Agricultural Science 39 (1991) 


\section{A. J. OSKAM}

Oskam, A. J., 1987. WASmodel. (In Dutch). Wageningse Economische Studies 1, Wageningen Agricultural University, Wageningen, $74 \mathrm{pp}$.

Oskam, A. J., 1991. Productivity measurement, incorporating environmental effects of agricultural production. In: K. Burger et al. (Eds), Agricultural economics and policy: international challenges for the nineties, p. 186-204. Elsevier, Amsterdam.

Oskam, A. J. \& A. J. Reinhard, 1992. Weather indices for agricultural production in the Netherlands 1948-1989. 2. Grassland and total agriculture. Netherlands Journal of Agricultural Science 40 (in press).

Oskam, A., A. Reinhard \& G. Thijssen, 1989. WASmodel-2: A disaggregated agricultural sector model partly based on prior information. In: S. Bauer \& W. Henrichsmeyer (Eds). Agricultural Sector Modelling, p. 53-69. Wissenschaftsverlag Vauk, Kiel.

Oury, B., 1965. Allowing for weather in crop production model building. Journal of Farm Economics 47: $250-282$.

Oury, B., 1966. A production model for wheat and feedgrains in France. North-Holland Publishing Company, Amsterdam, 306 pp.

Shaw, L. H., 1964. The effect of weather on agricultural output: a look at methodology. Journal of Farm Economics 46: 218-230.

Stallings, J. L., 1960. Weather indices. Journal of Farm Economics 42: 180-186.

Stallings, J. L., 1961. A measure of the effect of weather on crop production. Journal of Farm Economics 43: 1153-1160.

Thijssen, G. J., 1989. Production theory and dairy farms. (In Dutch). Wageningse Economische Studies 14. Wageningen Agricultural University, Wageningen, $95 \mathrm{pp}$.

Velde, H. A. te, 1984. Constraints on maize production in northern latitudes. In: E. J. Gallagher (Ed.), Cereal Production, p. 325-341. Butterworth, London.

Weber, A. \& M. Sievers, 1985. Instability in world food production: statistical analysis, graphical presentation and interpretation. Wissenschaftverlag Vauk, Kiel, $154 \mathrm{pp}$.

Wit, C. T. de, 1988. Environmental impact of the CAP. European Review of Agricultural Economics 15: 283-296. 